\title{
Review
}

\section{Role of proteases in activation of apoptosis}

\author{
Sergey A. Sukharev ${ }^{1,2}$, Olga V. Pleshakova ${ }^{1}$ and \\ Vladimir B. Sadovnikov ${ }^{1}$ \\ 1 Branch of Shemyakin and Ovchinnikov Institute of Bioorganic Chemistry, \\ Russian Academy of Sciences, 142292 Pushchino, Moscow region, Russia \\ 2 corresponding author: S.A. Sukharev, Branch of Shemyakin and Ovchinnikov \\ Institute of Bioorganic Chemistry, Russian Academy of Sciences, 142292 \\ Pushchino, Moscow region, Russia. tel: 7-095-9252345; fax: 7-095-9240493
}

Received 23.7.96; revised 26.3.97; accepted 7.4.97

Edited by C.J. Thiele

\begin{abstract}
Programmed cell death, or apoptosis, is a physiological cell suicide mechanism, which is triggered in the cells by different stimuli. It has been shown that proteases play a significant role both in the target cell killing by cytotoxic lymphocytes and in the TNF- or anti-Fas-induced cell death. The proteases involved in the early (induction) and late (cell self-destruction) stages of apoptosis are reviewed. It is suggested that the late stages are connected with the activation of a cascade of intracellular proteases, which leads to massive protein destruction. It is likely that the protein destruction is mainly designed for preventing autoimmune response to proteins released from dying cells.
\end{abstract}

Keywords: proteases, programmed cell death, apoptosis, targets of proteases

Abbreviations: CTL, cytotoxic lymphocytes; DCI, 3,4 dichloro isocoumarin; ICE, interleukin-1b converting enzyme or caspase-1; IL-1, interleukin-1; PARP, poly(ADP-ribose) polymerase; PMSF, phenylmethanesulphonyl fluoride; SOD, superoxide dismutase; TNF, tumor necrosis factor; TLCK, $\mathrm{N}$-tosyl-I-lysyl chloromethylketone; TPCK-N, tosyl-I-phenylalanine chloromethyl ketone; YVAD-CHO, N-(N-acetyl-tyrosinylvalinyl-alanyl-aspartyl)-3-amino-4-oxobutanoic acid; YVADCMK, (N-acetyl-tyrosinyl-valinyl-alanyl-aspartyl)-chloromethyl ketone

\section{Phenomenon and pathways of activation of apoptosis}

Apoptosis, the physiological mode of cell death, is representative of an endogenous cell suicide mechanism, which can be selectively triggered by cells in response to as yet largely unknown stimuli. It is an essential element of normal development of an organism. Apoptosis is observed during embryonic or fetal development or in cell turnover in normal adult tissue. In contract to necrosis, the cell actively participates in the process of its own death during apoptosis. The phenomenon of programmed cell death was described for the first time by Lockshin and Williams (1964), Whitfield et al (1968), Lockshin (1969). This mode of cell death was recognized as being separate from other forms of cell death and defined as apoptosis by Kerr et al (1972) and Wyllie (1980).

Apoptotic cells have specific biochemistry and morphology. The cell rounds up, severing junctions with its neighbours and losing microvilli. At the same time, the cytoplasm condenses, and the endoplasmic reticulum dilates, probably as a result of a net shift of water from the cytoplasm, and forms the vesicles, giving a characteristic bubbling appearance. The chromatin rapidly forms dense crescent-shaped aggregates lining the nuclear membrane (Duvall and Wyllie, 1986). One of the most well studied biochemical hallmarks of apoptosis is activation of $\mathrm{Ca}^{2+}, \mathrm{Mg}^{2+}$-nuclease, which causes the internucleosomal cleavage of nuclear chromatin. This results in appearance of low-molecular DNA fragments, whose size varies proportionally to the size of a nucleosome. When separated by electrophoresis, these fragments form a typical 'ladder'. The process of DNA degradation has been shown to involve three discrete stages: initially, to fragments of approximately $300 \mathrm{~kb}$, followed by the appearance of $50 \mathrm{~kb}$ fragments, and, finally, formation of low-molecular oligonucleosomal fragments (Brown et al, 1993).

The mechanisms of activation of apoptosis and, in particular, of endonucleases are still unknown. As it has been shown in many models of apoptosis, a proteolytic step is required prior to DNA cleavage by endonucleases (Bruno et al, 1992; Kaufman, 1989; Squier et al, 1994). Therefore, proteases seem to play a principal role in initiating the programmed cell death. Apoptosis may be induced by exogenous proteases injected into a cell (external activation pathway) or triggered upon activation of intracellular proteases by certain stimuli (internal activation pathway). The first type is mediated by cytotoxic lymphocytes, whose granules contain perforin and a variety of serine proteases - granzymes. Cell death induced by TNF, antibodies to Fas, or dexamethasone belongs to the second type. The mechanism is probably the same for the internal and external activation pathways. Injection of granzymes into the target cell may cause activation of endogenous proteases involved in the internal activation pathway.

\section{Granzymes and cytotoxicity of lymphocytes, or external activation pathway of apoptosis}

Cytotoxic $\mathrm{T}$ lymphocytes (CTL) destroy target cells by a mechanism involving membrane permeabilization and induction of apoptosis. During killing, their granules are exocytosed, releasing their contents into the intercellular space (Henkart, 1985). The CTL granules contain perforin and a variety of 
proteases - granzymes (A to $\mathrm{G}$ ). In the presence of $\mathrm{Ca}^{2+}$, perforin binds to the cell membrane, penetrates its lipid bilayer, and polymerizes, forming a pore approximately $16 \mathrm{~nm}$ in diameter (Podack et al, 1989), which facilitates the entry of proteolytic enzymes into the target cell.

Murine granzymes (specific cytotoxic proteinases) represent a gene family of seven members. They were originally identified as cytotoxic lymphocyte-specific genes, whose expession correlated with the induction of cytotoxicity (Lobe et al, 1986). Their localization within the lymphocyte cytoplasmic granules has been established (Redmond et al, 1987). The PCR analysis of granzymes $B-G$ in various $T$-cell activation models revealed that only granzyme B expression was correlated with the development of cytotoxicity in these cells (Prendergast et al, 1992), suggesting that it may be a direct effector in the lytic process. Shi et al (1992) have shown that fragmentin-2 - a serine protease purified from cytotoxic granules of rat natural killers and highly homologous to granzyme B - is able to induce DNA fragmentation and apoptosis in target cells in the presence of perforin. Perforin alone failed to trigger apoptosis. The importance of perforin and granzyme B was confirmed by subsequent studies. Stably transfected antisense constructs for perforin and granzyme B essentially eliminated the lytic ability of a human cytotoxic lymphocyte (Bochan et al, 1995). These results strongly indicate that both perforin and granzyme $B$ are required by this human cytotoxic lymphocyte for effective lysis. Recently the role of granzyme B in activation of apoptotic pathways was reviewed by Greenberg (1996).

Evidence also implicates granzyme $A$ in the induction of apoptosis. The expression of antisense granzyme A mRNA significantly decreased the cytotoxicity of transfectant cytotoxic lymphocytes (Talento et al, 1992). Moreover, the cotransfection of perforin and granzyme $A$ into a noncytotoxic basophil leukemia cell line (RBL) endowed this cell line with the capacity to induce apoptosis in target cells (Shiver et al, 1992). However, the data on the role of granzyme $A$ in induction of apoptosis are rather controversial. Thus, Ebnet et al (1995), generated granzyme Adeficient mouse mutants. Their in vitro- and ex vivo-derived cytotoxic $T$ cells and natural killer cells were indistinguishable from those of normal mice in causing membrane disruption, apoptosis and DNA fragmentation in target cells. The authors suggest that granzyme $A$ does not play a primary role in cell-mediated cytotoxicity, as has been assumed previously. The significance of proteases in activation of apoptosis has also been demonstrated in several studies using protease inhibitors. Thus, it has been shown that the cytotoxicity of cytotoxic lymphocytes and natural killers is strongly reduced by serine protease inhibitors present in the incubation medium (Chang et al, 1980; Hudig et al, 1981). Serine protease inhibitors also decrease the lytic activity of the granules isolated from cytotoxic lymphocytes (Hudig et al, 1992) and of purified serine protease preparations enriched with perforin (Ewoldt et al, 1992). Although the great deal of experimental data counts in favour of a key role of granzymes in cytotoxic lymphocyte-mediated apoptosis of target cells, the mechanism by which granzymes trigger target cell death and their specific intracellular substrates have not been completely established.

\section{Activation of intracellular proteases during apoptosis, or internal activation pathway}

This type of apoptosis is most often studied on the models of cell death induced by humoral factors-steroid hormones, TNF, anti-Fas antibodies.

Considering the similar apoptotic character of cell death triggered in a target cell by injection of CTL-derived granzymes or by physiological stimuli, Williams and Henkart (1994) examined the effects of cytoplasmic proteolysis on cells by using the techniques of osmotic lysis of pinosomes to introduce different proteases into cells. Such protease injection caused rapid cell death accompanied by DNA fragmentation and typical apoptotic morphological changes. These results suggest that activation of intracellular proteases by any molecular pathway could give rise to apoptotic cell death.

The increased protein degradation during the programmed cell death was detected by MacDonald et al $(1980,1981)$ in dexamethasone-treated lymphocytes. Voelkel-Johnson et al (1995) directly demonstrated the enhancement of lysis of several proteins in apoptotic cells. They measured proteolysis in the supernatants obtained after precipitation of total cell protein with $5 \%$ TCA by reaction of free primary amines with fluorescamine or by release of radioactively labeled leucine. Both the methods detected the increase in proteolytic activity in TNF-treated 3T3 cells.

Specific inhibitors have become an important tool in elucidating the role of proteases in apoptosis. It should be noted that chemically derived protease inhibitors may introduce artifacts related to their entry and distribution within the cell. Thus, Higuchi et al (1995) observed that two serine protease inhibitors abrogated the TNF-induced fragmentation of DNA and typical apoptotic morphological changes in a myelogenous cell line ML-1a; the maximum effect being observed in the presence of TPCK (Tos-Phe-CH2Cl) - a hydrophobic inhibitor of chymotrypsin-like proteases. However, hydrophobic compounds may interact with the membrane or membrane proteins. Therefore, it is not improbable that the effect of TPCK may be due to its influence, for instance, on the activity of phospholipase A2, which is known to be involved in the toxic action of TNF (Neale et al, 1988), but not to its protease-inhibiting properties.

Kwo et al (1995) have reported that TLCK - an inhibitor of trypsin-like serine proteases - is effective in preventing bile acid-induced DNA fragmentation in hepatocytes. However, other inhibitors of serine and cysteine proteases were ineffective in these cells. It should be noted that accurate comparison of effects of different protease inhibitors is complicated, since their efficiency may depend upon such factors as their ability to penetrate into the cell (which is not reported for the inhibitors used) or their stability (e.g. the stability of TLCK in water solution at physiological $\mathrm{pH}$ is very low). At the same time, one should keep in mind that the specificity of different protease inhibitors is not absolute. For instance, many serine protease inhibitors also inhibit cysteine proteases and vice versa. Chow et al (1995) have shown that 
cell lysates prepared from anti-Fas stimulated Jurkat $T$ cells induced chromatin fragmentation in isolated thymocyte nuclei. The formation of such activity in Jurkat T cells was almost completely blocked by the inhibitors of serine proteases as well as those of ICE. Therefore, the use of protease inhibitors as the only tool does not allow to elucidate the type of proteases involved in the programmed cell death unambiguously.

In spite of the rapidly growing interest in the model of Fasinduced apoptosis, its mechanism is at present unclear. The Fas (Apo-1/CD95) antigen is a cell surface receptor belonging to the TNF family. It is a $48 \mathrm{kDa}$ transmembrane glycoprotein (Oehm et al, 1992), and its cytoplasmic domain contains a region of significant sequence homology with the TNF receptor p55 (Itoh et al, 1991).

To date, it has been shown that one of the mediators of this activation mechanism is ICE - interleukin 1-bconverting enzyme or caspase-1. ICE is a cytoplasmic protease originally found in monocytes, which cleaves the IL-1b precursor $(31 \mathrm{kDa})$ with formation of the mature IL-1b $(17 \mathrm{kDa})$. Overexpression of ICE causes apoptosis of fibroblasts (Miura et al, 1993). Moreover, overexpression of Ced-3 - a protein from Caenorhabditis elegans, which belongs to the ICE family - also induced apoptosis (Yuan et al, 1993). Recently, the increase in proteolytic activity of ICE has been demonstrated with the use of its specific fluorescent substrate - DABCYL-YVADAP-EDANS. A marked hydrolysis of the substrate recorded by the increase in fluorescence was observed already within first $10 \mathrm{~min}$ after addition of anti-Fas antibodies (Los et al, 1995). As low as $10 \mathrm{nM}$ of a specific ICE inhibitor tetrapeptide aldehyde YVAD-CHO (Km=0.76 $\mathrm{nM})$ - almost completely inhibited the Fas-induced cell death. The transient transfection of cells with ICE constructs considerably increased the number of apoptotic cells, whereas CrmA (CrmA is a virus protein inhibitor of ICE) or ICE antisense constructs had the opposite effect (Los et al, 1995). These data are well consistent with those obtained by Enarl et al (1995) who have shown that an ICE inhibitor YVAD-chloromethylketone (YVAD-CMK) blocks cell death, although at much higher concentrations than YVAD-CHO. In addition, the authors have demonstrated the ability of the virus protein CrmA to prevent the TNF- or anti-Fasmediated cell death. The Rat-2 (rat fibroblast) transformants which expressed only human Fas were efficiently killed by TNF or anti-Fas. However, the transformant clones that expressed $\mathrm{CrmA}$ were resistant to such treatment.

Therefore, proteases play a key role in activation of apoptosis by both activation pathways. The proteolytic activity of several cellular proteases is increased as supported by the experiments with protease inhibitors. The activation of proteases causes degradation of several cell proteins, thus resulting in apoptotic biochemical and morphological changes.

\section{Specificity of proteases involved in apoptosis}

The important role of serine proteases - a family of proteolytic enzymes with serine in their active center - in induction of apoptosis has been well documented. Most granzymes are classified as serine proteases. According to their substrate specificity, the serine proteases may be divided into three large subgroups. Elastase-like enzymes cleave their protein substrates at non-charged non-aromatic amino acid residues (alanine, valine, glycine, etc.). Trypsin-like proteases (tryptases) cleave at positively charged amino acids (lysine, arginine). Chymotrypsin-like proteases (chymases) cleave at aromatic amino acid residues. There are also highly specific enzymes, which cleave a protein molecule at a single amino acid. For instance, granzyme $B$ is an asparaginase, since it cleaves only at asparagine.

The experiments made with the use of serine protease inhibitors support their dominant role in various models of apoptosis. Walker and Sikorska (1993) showed that several serine protease inhibitors (PMSF, DCI, TPCK) inhibited DNA fragmentation in thymocytes, whereas cysteine protease inhibitors (leupeptin, E64) did not. The cytoplasmic overexpression of a high-molecular serine protease inhibitor prevented the TNF-induced apoptosis (Kumar and Baglioni, 1991). In the above cited work by Chow et al (1995), the inhibitors of serine proteases and ICE were equally effective in inhibiting the formation of apoptotic nuclei promoting activity in anti-Fas treated Jurkat T cells, but only a specific chymase inhibitor (TPCK) was able to prevent the apoptosis in the isolated nuclei treated with the Jurkat $T$ cell extracts. These data also suggest that chymase, which immediately induces DNA fragmentation, itself must be activated by other proteolytic enzymes, probably, of different families, as demonstrated by the use of protease inhibitors with different specificity. This implies the existence of a cascade of proteases involved in activation of apoptosis in a cell. The significance of chymases in apoptosis is also supported by the experiments of Woodard et al (1994) who found that a specific biotinylated chymase inhibitor - Bi-Aca-Aca-Phe-Leu-Phe $P$ (Oph)2, which irreversibly binds to chymases, inhibited natural killer-mediated target cell lysis in a dose-dependent manner. Using the Western blot analysis, the authors identified a single $30 \mathrm{kDa}$ protein capable to bind to this inhibitor.

Although a great number of publications on the role of serine proteases have been accumulated, only few were successful in identifying individual enzymes. Schlegel et al (1995) partially purified a protease from the cytoplasm of Jurkat $T$ cells undergoing anti-Fas induced apoptosis and clearly demonstrated its belonging to the serine protease family. A $24 \mathrm{kDa}$ elastase-like serine protease has been purified from apoptotic U937 cells (Wright et al, 1994). The activity of this enzyme was more than tenfold higher in the apoptotic cells as compared to the control ones. In addition, the enzyme was able to trigger DNA fragmentation in isolated U937 nuclei.

Among the cysteine proteases, the role of ICE has been extensively studied. ICE is an asparaginase, i.e. it cleaves a protein molecule at asparagine. Asparaginases are relatively uncommon enzymes. Apart from ICE, only granzyme B, which belongs to the serine protease family, has been known among asparaginases related to apoptosis. However, granzyme B is not able to cleave the 
IL-1b precursor (Irmler et al, 1995), which indicates its higher specificity. Several other ICE-like proteases participating in activation of apoptosis have been described in many cells. One of the most widely known ICE-like proteins is CPP32 or caspase-3 (Fernandes et al, 1994). This protein has been cloned from Jurkat $T$ cells and found to be highly expressed in cell lines of lymphocytic origin. Overexpression of CPP32 or ICE in Sf9 cells caused the development of some apoptotic features in them. However, there are two possible interpretations of this and similar experiments on artificial overexpression of proteases in the cells. One is that the given protease is a part of endogenous death pathway, and its overexpression specifically activates the programmed cell death. Another possibility is that the death of a cell results from significant non-physiological protein destruction by overexpressed proteases. A protein of the ICE family similar to CPP32 has been described by Tewari et al (1995b). Although ICE has been found both in mice and in humans (Nett-Fiordalisi et al, 1992; Cerretti et al, 1994), CPP32 analog has not been discovered in mice. In mice, the product of the gene nedd2 - a cysteine protease, which is an inductor of apoptosis (Kumar et al, 1994), - has been described. This protein has a $25 \%$ homology to the human CPP32 (which is maximum as compared to its homology with other cysteine proteases), whereas the homology between the murine and human ICEs is about $60 \%$.

\section{Protein targets for proteases and a hypothetical mechanism of cell death}

In view of the foregoing, we can conclude that there exists a cascade of proteases involved in activation of apoptosis in the cell. Its enhancement with exogenously added proteases or inhibition by protease inhibitors would modulate the activation of programmed cell death. However, it seems necessary to distinguish between early and late, or advanced, stages of apoptosis. The early stage is characterized by initiation of the cascade of proteases-activation of certain cellular proteases and may be inhibited by highly specific inhibitors. The ability of specific inhibitors of ICE-like proteases to block apoptosis in different kinds of organisms suggests the central and conservative role of these proteases in apoptosis.

Little is known about the mechanism of activation of ICE. Chinnaiyan et al (1995) identified a protein FADD, which was able to bind to the cytoplasmic domain of Fas. Overexpression of FADD in MCF-7 or BJAB cells induced their apoptosis, which, similarly to Fas-activated apoptosis, was blocked by a specific ICE inhibitor CrmA. Therefore, FADD is likely an intermediate between Fas and ICE. Recently Boldin et al (1996) cloned a novel ICE/CED-3 protease, $\mathrm{MACH}$ or caspase-8, which binds to FADD. Its activity appears to be required for FAS and p55-R-induced cytotoxicity. It is likely caspase-8 is enzyme responsible for activating a protease cascade after Fas-receptor ligation, leading to death. However, the mechanisms of activation of the proteases by receptors have not yet been established.

Because of its secretory nature, the interleukin 1 precursor, which is cleaved by ICE, is unlikely to play a noticeable role in apoptosis. One of the targets for ICE-like proteases is poly(ADP-ribose) polymerase (PARP) (Tewari et al, 1995b). PARP is involved in repairing damaged DNA, therefore, its proteolytic inactivation accelerates apoptosis. The authors cloned an ICE-related gene. The protein thus obtained cleaved PARP to generate the $85 \mathrm{kDa}$ fragment typically found during apoptosis. Cleavage of PARP by this protein was inhibited by CrmA, but not by an inactive point mutant of CrmA. Furthermore, CrmA blocked cleavage of PARP in the dying cells. These experiments prove that PARP - a DNA-repairing enzyme - is one of the primary targets for ICE-like proteases.

One of the targets for granzyme $B$ is an intracellular cysteine protease CPP32, which belongs to the ICE family. Darmon et al (1995) have shown that granzyme B cleaves and activates CPP32, the precursor of the protease responsible for cleavage of poly(ADP-ribose) polymerase. It is possible that PARP is not the single target for CPP32. Therefore, entering the target cell, granzyme B may activate the intracellular cascade of proteases. The ICElike proteases are its key elements, which link (unite) external and internal pathways of apoptosis activation.

Using cell-free extracts, Lazebnik et al (1995) have shown that cleavage of the nuclear lamin during apoptosis requires the action of a second ICE-like enzyme, which exhibits kinetics of cleavage and a profile of sensitivity to specific inhibitors that is distinct from the PARP proteinase. Thus, multiple ICE-like enzymes are required for apoptotic events. Inhibition of the lamin proteinase with tosyllysine 'chloromethyl ketone' blocks nuclear apoptosis prior to the packaging of condensed chromatin into apoptotic bodies. Under these conditions, the nuclear DNA is fully cleaved to a nucleosomal ladder. The authors conclude that the lamin proteinase and the fragmentation nuclease function in independent parallel pathways during the final stages of apoptotic execution.

Fragmentation of DNA may be a result of the proteolytic activation of endonucleases, as demonstrated by Chow et al (1995), who have found that the protease-containing cytoplasmic fraction from apoptotic cells induces fragmentation of DNA in isolated nuclei. It is likely that a serine protease belonging to the cascade of intracellular proteases migrates into the nucleus, where it activates nucleases.

The activated cascade of proteases cleaves a large number of intracellular substrates and, therefore, induces alteration of cell morphology characteristic of the late stages of apoptosis, and the addition of different protease inhibitors significantly reduces these morphological and biochemical changes. The rounding of the cells and their detachment from the substrate may result from destruction of the cytoskeleton. Indeed, Suarez-Huerta et al (1995) have demonstrated the degradation of cytoskeleton proteins during the TNF-induced apoptosis. CasciolaRosen et al (1995) observed the apoptosis-related destruction of DNA-dependent protein kinase. Tewari et al (1995a) observed the destruction of the $70 \mathrm{kDa}$ protein component of U1 small nuclear ribonucleoprotein during Fas- and TNF-induced apoptosis. Voelkel-Johnson et al (1995) have revealed degradation of several other proteins during apoptosis: topoisomerase, laminin $B$, histone $H 1$, protein kinase $\mathrm{C}$, phospholipase $\mathrm{A} 2$. It hardly can be 
suggested that all proteins are involved in signal pathway of apoptosis and degraded specifically. The targets of cysteine proteases and a scheme of their interaction during apoptosis have been described in details by Kumar and Lavin (1996). It seems most probably that several proteases are activated or, possible, lysosomal enzymes are released as a result of cytoskeleton destruction. So Lockshin and Zaker (1994) observed the lysosomal destruction of cytoplasm at apoptosis of intrasegmental muscles and labial glands of Manduca sexta.

Since in most apoptotic models inhibitors of transcription and translation induce (Martin et al, 1990) or enhance apoptosis in dose-dependent manner (Voelkel-Johnson et al, 1995; Higuchi et al, 1995), it is most probable that the cascade of proteases already exists in the cell and is not synthesized de novo. Activation of apoptosis leads to formation of active enzymes from their precursors. There may be an equilibrium maintained in the cell between degradation of proteins by proteases and their biosynthesis. Introduction of exogenous or activation of endogenous proteolytic enzymes as well as addition of inhibitors of protein synthesis may shift this equilibrium to the acceleration of protein degradation. The concentrations of physiologically active proteins are then decreased, resulting in a considerable metabolic injury: e.g. impairment of DNA repair by PARP, destruction of the cytoskeleton, presumably, loss of antioxidant defense (SOD etc.), alteration of the chromatin structure (histones), etc. Taken together, these damages cause the cell death.

Therefore, the early stages of activation of apoptosis are initiated by individual proteases, most probably, ICE-like enzymes, which are inhibited by highly specific protease inhibitors. ICE-like proteases activate an intracellular protease cascade. The ICE-activated proteases cascade destroy or activate various cell proteins. Thus, destruction of cytoplasmic proteins gives the typical apoptotic morphology. Proteolytic cleavage of endonucleases causes their activation followed by degradation of DNA. Therefore, at the late stages, the functioning of the whole cascade of cellular proteases is accompanied by non-specific destruction of several cellular proteins and leads to the cell death. It is likely that the protein destruction is mainly designed for preventing the immune response to intracellular proteins released from the cell after its disintegration. The antigenicity of proteins is decreased by proteolytic fragmentation before the cell membrane breakage.

\section{Acknowledgements}

We thank Russian Foundation for Fundamental Research for support.

\section{References}

Bochan MR, Goebel WS and BrahmiZ (1995) Stably transfected antisense granzyme $B$ and perforin constructs inhibit human granule-mediated lytic ability. Cellular Immunology 164: 234-239

Boldin MP, Goncharov TM, Goltsev YV and Wallach D (1996) Involvement of MACH, a novel MORT1/FADD-interacting protease, in Fas/APO-1- and TNF receptorinduced cell death. Cell 85: 803-815
Brown DG, Sun XM and Cohen GM (1993) Dexamethasone induced apoptosis involves cleavage of DNA to large fragments prior to internucleosomal fragmentation. J. Biol. Chem. 268: 3037-3039

Bruno S, Del Bion G, Lassota P, Giaretti W and Darzynkiewicz Z (1992) Inhibitors of proteases prevent endonucleolysis accompanying apoptotic death of $\mathrm{HL}-60$ leukemic cells and normal thymocytes. Leukemia 6: 1113-1120

Casciola-Rosen LA, Anhalt GJ and Rosen A (1995) DNA-dependent protein kinase is one of subset of autoantigens specifically cleaved early during apoptosis. J. Exp. Med. 182: 1625-1634

Cerretti DP, Hollingsworth LT, Kozlosky CJ, Valentine MB, Shapiro DN, Morris SW and Nelson N (1994) Molecular characterization of the gene for human interleukin-1b converting enzyme. Genomics 20: 468-473

Chang TW and Eisen HN (1980) Effects of N-tosyl-L-lysyl-chloromethylketone on activity of CTL. J. Immunol. 124: 1028-1032

Chinnaiyan AM, O'Rourke K, Twari M and Dixit VM (1995) FADD, a novel death domain containing protein, interacts with death domain of FAS and initiate apoptosis. Cell 81: $505-512$

Chow SC, Weis M, Kas GEN, Holmstrom TH, Erikson JE and Orrenius S (1995) Involvement of multiple proteases during FAS mediated apoptosis in T lymphocytes. FEBS Lett. 364: 134-138

Darmon AJ, Nicholson DW and Bleackley RC (1995) Activation of the apoptotic protease CPP32 by cytotoxic T-cell-derived granzyme B. Nature 377: 446448

Duvall E and Wyllie AH (1986) Death and the cell. Immunol. Today 7: 115-119

Ebnet K, Hausmann M, Lehmanngrube F, Mullbacher A, Kopf M, Lamers M and Simon MM (1995) Granzyme A-deficient mice retain potent cell-mediated cytotoxicity. EMBO 14: 4230-4239

Enarl M, Hug H and Nagata S (1995) Involvement of ICE-like proteases in Fasmediated apoptosis. Nature 375: 78-81

Ewoldt GR, Winkler U, Powers JC and Hudig D (1992) Sulfonyl fluoride serine protease inhibitors inactivate RNK-16 lymphocytes granule proteases and reduce lysis by granule extracts and perforin. Mol. Immunol. 29: 713-721

Fernandes-Alnemri T, Litwack G and Alnemri ES (1994) CPP32, a novel human apoptotic protein with homology to Caenorhabditis elegans cell death protein Ced-3 and mammalian interleukin-1b-convertnig enzyme. J. Biol. Chem. 269: $30761-30764$

Greenberg A (1996) Activation of apoptotic pathways by granzyme B. Cell Death Differ. 3: 269-274

Henkart PA (1985) Mechanism of lymphocyte-mediated cytotoxicity. Annu. Rev. Immunol. 3: 31-58

Higuchi M, Singh S, Chan H and Aggarwal B (1995) Protease inhibitors differentially regulate tumor necrosis factor induced apoptosis, nuclear factor-kappa $B$ activation, cytotoxicity and differentiation. Blood 86: 2248-2256

Hudig D, Allison NJ, Pickett TM, Winkel U, Kam CM and Powers JC (1992) The function of lymphocytic proteases: inhibition and restoration of granule-mediated Iysis with isocoumarin serine proteases inhibitors. J. Immunol. 147: 1360-1364

Hudig D, Haverty T, Fulcher C, Redelman D and Mendelsohn J (1981) Inhibition of human natural cytotoxicity by macromolecular antiproteases. J. Immunol. 126: $1569-1575$

Irmler B, Hertig S, McDonald HR, Sadoul R, Becherer JD, Proudfoot A, Solari R and Tschopp J (1995) Granzyme A is an interleukin 1-converting enzyme. J. Exp. Med. 181: 1917-1922

Itoh N, Yonehara S, Ishii A, Yonehara M, Mizushima S, Sameshima M, Hase A, Seto Y and Nagata $S$ (1991) The polypeptide encoded by the cDNA for human cell surface antigen Fas can mediate apoptosis. Cell 66: 233-243

Kaufmann S (1989) Induction of endonucleolytic DNA cleavage in human acute myelogenous leukemia cells by etoposide, camptothecin, and other cytotoxic anticancer drugs: a cautionary note. Cancer Research 49: $5870-5878$

Kerr JFR, Wyllie AH and Currie AR (1972) Apoptosis: a basic biological phenomenon with wide ranging implications in tissue kinetics. British Journal of Cancer 26: $239-257$

Kumar S and Baglioni C (1991) Protection from tumor necrosis factor mediated cytolysis by overexpression of plasminogen activator inhibitor type-2. J. Biol. Chem. 266: 20960-20965

Kumar S, Kinoshita M, Noda M, Copeland NG and Jenkins NA (1994) Induction of apoptosis by mouse Nedd2 gene which encodes a protein similar to the Caenorhabditis elegans cell death gene ced-3 and mammalian IL-1b-converting enzyme. Genes Dev. 8: 1613-1626 
Kumar Sand Lavin MF (1996) The ICE family of cysteine proteases as effectors of cell death. Cell Death Differ. 3: 255-267

Kwo P, Patel T, Bronk SF and Gores GJ (1995) Nuclear serine protease activity contributes to bile acid-induced apoptosis in hepatocytes. Am. J. Physiol. 268: G613-G621

Lazebnik YA, Takahashi A, Moir RD, Goldman RD, Poirier GG, Kaufmann SH and Earnshaw WC (1995) Studies of the lamin proteinase reveal multiple parallel biochemical pathways during apoptotic execution. Proceedings of the National Academy of Sciences of the United States of America 92: 9042-9046

Lobe CG, Havele C and Bleackley RC (1986) Cloning of two genes that are specifically expressed in activated cytotoxic T lymphocytes. Proceedings of the National Academy of Sciences of the United States of America 83: 1448-1452

Lockshin RA (1969) Programmed cell death. Activation of lysis by mechanism involving the synthesis of protein. J. Insect. Physiol. 15: 1505-1516

Lockshin RA and Williams CM (1964) Programmed cell death. Endocrine potentiation of the breakdown of the intersegmental muscles of silkmoths. J. Insect. Physiol. 10: $643-649$

Lockshin RA and Zaker Z (1994) Programmed cell death: early changes in metamorphosis cells. Biochem. Cell Biol. 72: 589-599

Los M, de Craen MV, Penning LC, Schenk H, Westendorp M, Baeuerle M, Droge W, KrammerPH, Flers W and Shulze-Osthoff K (1995) Requirement of an ICE/CED3 protease for Fas/APO-1 mediated apoptosis. Nature 375: 81-83

MacDonald RG and CidlowskiJ (1981) Glucorticoids-stimulated protein degradation in lymphocytes: quantitation by SDS-gel electrophoresis. Arch. Biochem. Biophys. 212: $399-410$

MacDonald RG, Martin TS and Cidlowski JA (1980) Glucorticoids stimulate protein degradation in lymphocytes: possible mechanism of steroid induced cell death. Endocrinology 107: 1512-1524

Martin SJ, Lennon SV, Bonham AM and Cotter TG (1990) Induction of apoptosis in human leukemic cells by inhibition of RNA or protein synthesis. J. Immunol. 90: $1859-1867$

Miura M, Zhu H, Rotello R, Hartwieg EA and Yuan J (1993) Induction of apoptosis in fibroblasts by IL-1b converting enzyme, a mammalian homolog of the $\mathrm{C}$. elegans cell death gene ced-3. Cell 75: 653-660

Neale ML, Fierra RA and Matthews D (1988) Involvement of phospholipase $A_{2}$ activation in tumor cell killing by TNF. Immunology $64: 81-85$

Nett-Fiordalisi MA, Cerretti DP, Berson DR, Gilbert DJ, Jenkins NA, Copeland NG Black RA and Chaplin DD (1992) Molecular cloning of the murine IL-1b converting enzyme cDNA. J. Immunol. 149: 3254-3259

Oehm A, Behrmann I, Falk W, Pawlita M, Maier G, Klaus C, Li-Weber M, Richard S, Dhein J, Trauth BC, Ponsting H and Kramer PH(1992) Purification and molecular cloning of the APO-1 cell surface antigen, a member of the tumor necrosis factor/ nerve growth factor receptor superfamily-sequence identity with the Fas. J. Biol. Chem. 267: 10709-10715

Podack ER, Olsen KJ, Lowrey DM, Lichtenheld M (1989) Structure and function of perforin. Curr. Top. Microbiol. Immunol. 140: 11-17

Prendergast JA, Helgason CD and Bleackley RC (1992) Quantitative polymerase chain reaction analysis of cytotoxic cell proteinase gene transcripts in T cells. J. Biol. Chem. 267: 5090-5095
Redmond MJ, Letellier M, Parker JM, Lobe C, Havele C, Paetkau V and Bleackley RC (1987) A serine proteases sequestered in the cytoplasmic granules of cytotoxic lymphocytes. J. Immunol. 139: 3184-3188

Squier M, Miller A, Malkinson A and Cohen J (1994) Calpain activation in apoptosis. J. Cell Physiol. 159: 229-237

Schlegel J, Peters I and Orrenius S (1995) Isolation and partial characterization of a protease involved in FAS-induced apoptosis. FEBS Lett. 364: 139-142

ShiverJM, Su L and HenkartPA (1992) Cytotoxicity with target DNA breakdown by rat basophilic leukemia cells expressing both cytolysin and granzyme A. Cell 71 315-322

Shi L, Kraut RP, Aebersold R and Greenberg AH (1992) A natural killer cell granule protein that induces DNA fragmentation and apoptosis. J. Exp. Med. 175: 553566

Suarez-Huerta N, Lecocq R, Mosselmans R, Dumont JE and Robaye B (1995) Specific degradation of cytoskeleton proteins during apoptosis. The third euroconference on apoptosis. 21-25 October, Cuenca, Spain, poster 71

Talento A, Nguyen M, Law S, Wu JK, Poe M, Blake JT, Patel M, Wu T, Manyak CL, Silberklang M, Mark G, Springer M, Sigal NH, Weissman IL, Bleackley RC Podack ER, Tykocinski ML and Koo GC (1992) J. Immunol. 149: 4009-4015

Tewari M, Beidler DR and Dixit VM (1995a) Crma-inhabitable cleavage of the $70 \mathrm{kDa}$ protein component of $\mathrm{U} 1$ small nuclear ribonuclearprotein during Fas- and TNFinduced apoptosis. J. Biol. Chem. 270: 18738-18741

Tewari M, Quan LT, O'Rourke K, Desnoyers S, Zeng Z and Beidler (1995b) Yama/ CPP32 beta, a mammalian homolog of CED-3, is a CrmA inhibitable protease that cleaves the death substrate poly(ADP-ribose) polymerase. Cell 81: $801-$ 809

Voelkel-Johnson C, Entigh AJ, William SM Wold, Gooding LR and Laster SM (1995) Activation of intracellular proteases is an early event in TNF-induced apoptosis. J. Immunol. 154: 1707-1716

Walker PR and Sikorska M (1993) Role of proteolysis in apoptosis: involvement of serine proteases in internucleosomal DNA fragmentation in immature thymocytes. Biochem. Cell Biol. 71: 488-500

Whitfield JF, Perris AD and Youdale T (1968) Destruction of the nuclear morphology of thymic lymphocytes by the corticosteroid cortisol. Exp. Cell. Res. 52: 349-362

Williams MS and Henkart PA (1994) Apoptotic cell death by intracellular proteolysis. J. Immunol. 153: 4247-4255

Woodard S, Jackson DS, Abuelaman AS, Powers JC, Winkler U and Hudig D (1994) Chymase-directed serine protease inhibitor that reacts with a single $30 \mathrm{kDa}$ granzyme and blocks NK-mediated cytotoxicity. J. Immunol. 153: 5016-5025

Wright SC, Wei QS, Zhong J, Zheng H, Kinder DH and Larrick JW (1994) Purification of a $24-\mathrm{kD}$ protease from apoptotic tumor cells that activates DNA fragmentation. J. Exp Med. 180: 2113-2123

Wyllie AH (1980) Glucorticoids induced thymocytes apoptosis is associated with endonuclease activation. Nature 284: $555-556$

Yuan J, Shalam S, Ledoux S, Ellis HM and Horvitz HR (1993) The C. elegans death gene encodes a protein similar to mammalian interleukin-1b converting enzyme. Cell 75: 641-652 